\title{
Qualidade de vida de pessoas soropositivas: relação entre situação socioeconômica e estágio viral
}

\section{Quality of life of HIV-positive people: relationship between socioeconomic status and viral stage}

\section{Calidad de vida de personas seropositivas: relación entre la situación socioeconómica y el estado viral}

\author{
João Ferreira Silva Junior iD \\ Universidade Federal do Maranhão - São Luís - (MA) - Brasil \\ Carlos Martins Neto iD \\ Universidade Federal do Maranhão - São Luís - (MA) - Brasil \\ Bruno Luiz Avelino Cardoso iD \\ Universidade Federal de São Carlos - São Carlos (SP) - Brasil \\ Elisa Miranda Costa iD \\ Universidade Federal do Maranhão - São Luís - (MA) - Brasil
}

Olga Lorena Maluf Guará Beserra (iD

Faculdade Santa Terezinha - São Luís - (MA) - Brasil

Verônica Silva Carneiro (iD

Universidade Ceuma - São Luís - (MA) - Brasil

\section{RESUMO}

Objetivo: Analisar a influência da situação socioeconômica e do estágio do vírus da imunodeficiência humana na qualidade de vida de pessoas soropositivas. Métodos: Trata-se de um estudo quantitativo e transversal, realizado entre 2016 e 2017, desenvolvido em um centro de referência no tratamento de pessoas com o vírus da imunodeficiência humana em São Luís (Maranhão, Brasil). A amostra compôs-se de 122 pessoas soropositivas que realizavam tratamento e acompanhamento nesse centro de referência. Utilizou-se um instrumento para coleta de dados socioeconômicos e o questionário de qualidade de vida WHOQol-HIV-bref. A análise dos dados ocorreu por meio da modelagem com equações estruturais. Construíram-se duas variáveis latentes, situação socioeconômica e qualidade de vida, utilizando análise fatorial confirmatória e, em seguida, a análise de caminhos. Resultados: Todas as cargas fatoriais das variáveis latentes apresentaram-se convergentes. Em relação à parte estrutural do modelo, para cada variação de um desvio padrão na situação socioeconômica, há um aumento de 0,601 desvio padrão na qualidade de vida dos participantes. Não houve efeito direto significante do estágio clínico do vírus na qualidade de vida $(p=0,165)$, nem da situação socioeconômica no estágio clínico $(p=0,610)$, bem como não houve efeito indireto da situação socioeconômica na qualidade de vida quando mediado pelo estágio clínico $(p=0,654)$. Conclusão: $O$ estágio clínico não se associou à qualidade de vida e não apresentou efeito mediador entre a situação socioeconômica e a qualidade de vida. A situação socioeconômica influenciou diretamente a qualidade de vida dos participantes.

Descritores: Classe Social; Síndrome da Imunodeficiência Adquirida; Soropositividade para HIV; Qualidade de Vida; Análise Estatística.

\section{ABSTRACT}

Objective: To analyze the influence of socioeconomic status and human immunodeficiency virus stage on the quality of life of HIVpositive people. Methods: This quantitative cross-sectional study was conducted between 2016 and 2017 at a reference center for the treatment of people with human immunodeficiency virus in São Luís (Maranhão, Brazil). The sample comprised 122 HIVpositive people undergoing treatment and follow-up at the reference center. We used an instrument to collect socioeconomic data and the WHOQol-HIV-bref. Data analysis was performed using structural equation modeling. Two latent variables were constructed - socioeconomic status and quality of life - using confirmatory factor analysis followed by path analysis. Results: All factor loadings

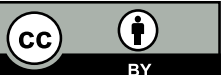


of the latent variables described converge. With regard to the structural part of the model, for each variation of one standard deviation in the socioeconomic status there was an increase of 0.601 standard deviation in the quality of life of the participants. The clinical stage of the virus had no significant direct effect on quality of life $(p=0.165)$. Also, socioeconomic status had no significant effect on the clinical stage of the virus ( $p=0.610)$. Additionally, socioeconomic situation did not have any indirect effect on quality of life mediated by clinical stage $(p=0.654)$. Conclusion: The clinical stage was not associated with quality of life and did not have a mediating effect on socioeconomic status and quality of life. The socioeconomic status directly influenced the participants' quality of life.

Descriptors: Social Class; Acquired Immunodeficiency Syndrome; HIV Seropositivity; Quality of Life; Statistical Analysis.

\section{RESUMEN}

Objetivo: Analizar la influencia de la situación socioeconómica y del estado del virus de la inmunodeficiencia humana en la calidad de vida de personas seropositivas. Métodos: Se trata de un estudio cuantitativo y transversal realizado entre 2016 y 2017 desarrollado en un centro de referencia para el tratamiento de personas con el virus de la inmunodeficiencia humana de São Luís (Maranhão, Brasil). La muestra ha sido de 122 personas seropositivas que realizaban el tratamiento y el seguimiento en ese centro de referencia. Se utilizó un instrumento para la recogida de datos socioeconómicos y el cuestionario de calidad de vida WHOQol-VIH-bref. El análisis de los datos se dio a través de ecuaciones estructurales. Se ha construido dos variables latentes, la situación socioeconómica y la calidad de vida, utilizándose el análisis factorial confirmatorio y después el análisis de caminos. Resultados: Todas las cargas factoriales de las variables latentes se presentaron convergentes. Respecto la parte estructural del modelo para cada variación de una desviación típica de la condición socioeconómica hay un aumento de 0,601 desviación típica de la calidad de vida de los participantes. No hubo el efecto directo significativo del estado clínico del virus para la calidad de vida $(p=0,165)$ ni de la situación socioeconómica del estado clínico $(p=0,610)$ así como no hubo el efecto indirecto de la situación socioeconómica en la calidad de vida cuando mediado por el estado clínico $(p=0,654)$. Conclusión: El estado clínico no se asoció con la calidad de vida y no presentó el efecto mediador entre la situación socioeconómica y la calidad de vida. La situación socioeconómica ha influenciado directamente en la calidad de vida de los participantes.

Descriptores: Clase Social; Síndrome de Inmunodeficiencia Adquirida; Seropositividad para VIH; Calidad de Vida; Análisis Estadístico.

\section{INTRODUÇÃO}

A epidemia brasileira do vírus da imunodeficiência humana/acquired immunodeficiency syndrome (HIVIAIDS) constitui um emaranhado de surtos regionais e locais de diferentes características em sua quarta década(1). Como resultado das profundas desigualdades da sociedade brasileira, a propagação da infecção pelo HIV no Brasil revela um flagelo de múltiplas dimensões, que vem, ao longo do tempo, sofrendo transformações significativas em seu perfil epidemiológico(2).

No ano de 2017 foram notificados 42.420 casos de infecção pelo HIV, sendo $9.706(22,9 \%)$ casos na região Nordeste. No Maranhão, houve um aumento de $69 \%$ na taxa de detecção da AIDS por 100 mil habitantes entre $2007(12,6 \%)$ e 2017 (21,3\%), constituindo o $10^{\circ}$ estado no ranking brasileiro ${ }^{(3)}$. A mortalidade por AIDS também aumentou $27 \%$ nesses 10 anos. Mesmo ainda sem cura, a infecção pelo HIV possui tratamento e é considerada uma condição crônica manejável para muitos indivíduos ${ }^{(4)}$.

O cuidado contínuo em HIV pode ser entendido como o processo de atenção às pessoas vivendo com HIV/ AIDS (PVHA). Esse método passa pelos seguintes momentos: diagnóstico oportuno; vinculação do indivíduo HIV positivo a um serviço de saúde; sua retenção no seguimento através do acompanhamento e da realização de exames periódicos; início da terapia antirretroviral (TARV) e sua promoção para uma boa adesão ao tratamento. Tudo isso é feito para alcançar os objetivos finais do cuidado e a supressão da carga viral, com alcance de uma qualidade de vida (QoL) comparável à das pessoas que não possuem o HIV(5).

Para o cuidado contínuo, utilizam-se medidas padronizadas de estado de saúde relatado pelo paciente, o qual inclui sintomas, estado funcional e QoL; oferecendo, assim, uma abordagem válida, sensível e reprodutível para manejar os resultados de saúde do ponto de vista do paciente( ${ }^{(6)}$.

A infecção pelo HIV apresenta-se com um amplo espectro de apresentações clínicas, desde a fase aguda até a fase avançada da doença. Em pessoas não tratadas, estima-se que o tempo médio entre o contágio e o aparecimento da doença esteja em torno de dez anos, compreendendo as fases de infecção aguda, latência clínica e AIDS ${ }^{(1)}$.

O conhecimento do paciente sobre seu status sorológico e estadiamento clínico é um fator preditor do sucesso no tratamento e na prevenção de complicações após a infecção. Atualmente, o tratamento mais eficaz para PVHA constitui-se na associação da TARV e no desenvolvimento de hábitos de vida saudáveis, como boa alimentação, exercícios físicos diários, relações sexuais com segurança e uso de preservativos, entre outros ${ }^{(7)}$. 
A partir de novas descobertas relacionadas ao HIVIAIDS, a configuração epidemiológica da doença, bem como suas características e das pessoas infectadas, sofreu modificações. Uma PVHA coexiste com particularidades devido ao processo de infecção pelo HIV e precisa de ajuda para manter-se estável, tanto com sua saúde como nas rotinas de vida(8).

O estágio do HIV era, no passado, um fator de risco para uma pior QoL de pessoas soropositivas ${ }^{(9)}$, no entanto novas opções de tratamento foram sendo desenvolvidas, o que aumentou a sobrevida e a melhora da QoL dos atingidos $^{(10)}$. Um conjunto de ações através de medicamentos conjugados ( 3 em 1 ) e com menos efeitos colaterais inseridos nos protocolos clínicos, que facilitam a aderência ao tratamento ${ }^{(11)}$, bem como o melhor acolhimento nas unidades de saúde ${ }^{(12)}$, possibilitou importantes resultados frente à pandemia global ${ }^{(13)}$.

Com base no exposto, o objetivo do presente estudo é analisar a influência da situação socioeconômica e do estágio do vírus da imunodeficiência humana na qualidade de vida de pessoas soropositivas.

\section{MÉTODOS}

Este é um estudo quantitativo e transversal, realizado entre outubro de 2016 e abril de 2017, no Centro de Saúde de Fátima, espaço no qual funciona o Serviço de Assistência especializada em HIVIAIDS, no município de São Luís (Maranhão, Brasil). São Luís é uma cidade pertencente ao Nordeste brasileiro, com uma ampla rede de prevenção, diagnóstico e tratamento em HIVIAIDS.

Participaram deste estudo usuários que faziam acompanhamento médico e com a equipe de Enfermagem no centro de saúde, compondo uma amostra não probabilística, que se deu por conveniência, de 122 participantes soropositivos para HIV. Incluíram-se indivíduos com idade entre 18 e 59 anos, com diagnóstico médico de HIV confirmado, de ambos os sexos, que estavam em acompanhamento no centro de saúde e que aceitaram participar voluntariamente da pesquisa assinando Termo de Consentimento Livre e Esclarecido. Não se incluiu no estudo os indivíduos com doenças crônicas e degenerativas, gestantes, menores de 18 anos e que se recusaram a participar da pesquisa.

Coletaram-se os dados por dois pesquisadores previamente treinados e acompanhados pela assistente social do centro de saúde. Não houve diferença significante entre os dados coletados entre os dois pesquisadores $(p>0,05)$.

Realizou-se a coleta de dados através de dois questionários. O primeiro incluiu questões relacionadas ao perfil socioeconômico e demográfico, além de questões sobre o nível de satisfação da saúde do indivíduo. Deste, utilizou-se a renda mensal e a escolaridade dos indivíduos nas análises. Não se realizou estudo piloto para esse questionário.

O segundo questionário é o de qualidade de vida WHOQol-HIV-bref, traduzido e validado no Brasil(14). A Organização Mundial de Saúde (OMS) desenvolveu esse questionário com o intuito de avaliar a QoL dentro de uma perspectiva internacional. O questionário WHOQol-HIV-bref divide-se em seis domínios e suas facetas: domínio I - físico (dor, desconforto, energia, qualidade do sono e repouso); domínio II - psicológico (sentimentos positivos e negativos, qualidade de aprendizagem, capacidade de memorização, autoestima e aparência); domínio III nível de independência (atividades da vida diária, mobilidade, dependência de tratamento médico e medicações e capacidade para o trabalho); domínio IV - relações sociais (relações pessoais, atividade sexual, apoio e inclusão social); domínio $\mathrm{V}$ - ambiente (moradia, segurança física, recursos financeiros, lazer e ambiente físico); domínio VI - espiritualidade/religiosidade/crenças pessoais (espiritualidade, religiosidade, crenças pessoais, preocupação com o futuro e medo da morte). Cada um desses instrumentos somou-se ao domínio particular da QoL em que o indivíduo está inserido.

Selecionaram-se as variáveis para os constructos situação socioeconômica e QoL após a análise fatorial confirmatória através de suas cargas fatoriais. A variável latente QoL é composta pelos domínios do questionário WHOQol-HIV-bref: domínios físicos, psicológico, independência funcional, relações sociais, meio ambiente e espiritualidade. Cada domínio é composto por questões específicas e os valores dos escores variaram de 4 a 20 , sendo que, quanto maior o escore, melhor a QoL ${ }^{(14)}$.

A variável latente situação socioeconômica compôs-se pela renda em salários, considerando o valor de 2017 (menos de um salário; $1 ; 1$ a $2 ; 2$ a $3 ; 3$ a $4 ; 4$ ou mais) e a escolaridade $\left(1^{\circ}\right.$ grau incompleto; $1^{\circ}$ grau completo; $2^{\circ}$ grau incompleto; $2^{\circ}$ grau completo; $3^{\circ}$ grau incompleto; $3^{\circ}$ grau completo e pós-graduação) conforme preconiza um estudo brasileiro(15).

Nas análises, estimaram-se as frequências absolutas e percentuais e os intervalos de confiança a 95\% (IC95\%) para as variáveis categóricas, bem como médias ( \pm desvios-padrão) ou medianas ( \pm desvios interquartílicos), respectivamente, para variáveis numéricas com distribuição simétrica e assimétrica. Para tal, utilizou-se o programa Stata, versão 14.0 (Stata Corp., College Station, United States). 
A modelagem de equações estruturais (MEE) é uma técnica para lidar com múltiplas relações de dependência simultaneamente e conseguir representar conceitos não observados nessas relações, reduzindo o erro de mensuração no processo de estimação. Essa análise estatística estima uma série de equações de regressão múltipla. $O$ modelo é um suposto padrão de relações lineares diretas e indiretas entre um conjunto de variáveis observadas e construtos. Este consiste em dois submodelos: o modelo de mensuração, que estabelece como os constructos são medidos; e o modelo estrutural, que analisa o modelo teórico como um todo, na qual as associações entre as variáveis são estimadas por cargas fatoriais padronizadas (CFP). As CFP do modelo estrutural foram interpretadas da seguinte forma: coeficientes com valores próximos a 0,10 indicam um efeito pequeno; próximos a 0,30, efeito médio; superiores a 0,50 indicam um efeito forte. Cargas fatoriais negativas indicam associação inversa e cargas positivas indicam associação direta ${ }^{(16)}$.

A elaboração de uma variável latente faz-se no modelo de mensuração, em que os indicadores da variável latente são especificados. Uma boa variável latente apresenta validade convergente, verificado pelas cargas fatoriais padronizadas com valores elevados (superior a 0,50). Além disso, verificou-se se a correlação entre dois construtos não seria maior que 0,90 (validade discriminante), ou seja, se os dois constructos deviam medir aspectos distintos. Cargas fatoriais negativas indicam associação inversa e cargas positivas indicam associação direta ${ }^{(17)}$.

Avaliou-se o modelo do estudo pelos índices de ajuste, incluindo-se o RMSEA (Root Mean Square Error of Aproximation), o CFI (Comparative Fit Index), o TLI (Tucker-Lewis Index) e o WRMR (Weighted Root Mean Square Residual). Consideram-se como valores de índice de ajuste aceitáveis para os modelos: RMSEA<0,05; os limites superiores do IC a 90\% do RMSEA<0,08; os valores de CFI e TLI >0,95; e de WRMR<1,00. Devido a não normalidade na distribuição dos dados, utilizou-se o método robusto MLM (máxima verossimilhança ajustada pela média). Estimou-se, através das CFP, o alpha de 5\%. Estimou-se o modelo proposto usando-se o software Mplus 8 (Inc., Los Angeles, United States).

A pesquisa obedeceu aos aspectos éticos e legais que aprovam diretrizes e normas para desenvolvimento de pesquisa com seres humanos, de acordo com a Resolução n. ${ }^{\circ} 466 / 12$ do Conselho Nacional de Saúde (CNS), sendo aprovada pelo Comitê de Ética em Pesquisa (CEP) do Hospital São Domingos com o Parecer consubstanciado n. ${ }^{0} 1.094 .120$.

\section{RESULTADOS}

Os participantes do presente estudo possuíam média de idade de 37,5 anos $( \pm 9,93)$, variando entre 18 e 58 anos, sendo $64(52,46 \%)$ do sexo feminino e $58(47,54 \%)$ do sexo masculino.

Quanto à origem, 56 (45,9\%) são da capital São Luís e 66 (54,1\%) provém do interior do estado (Tabela I).

Tabela I - Caracterização do estágio do HIV, escolaridade, renda e domínios da qualidade de vida. São Luís, Maranhão, Brasil, 2016-2017.

\begin{tabular}{|c|c|c|c|}
\hline Variável & Descrição & Codificação & Frequência (\%) \\
\hline estágio & Estágio do HIV & $\begin{array}{l}\text { 1: HIV positivo assintomático } \\
\text { 2: HIV positivo sintomático } \\
\text { 3: AIDS (doenças relacionadas) } \\
\text { 4: HIV negativo (indetectável) }\end{array}$ & $\begin{array}{c}57(46,72) \\
15(12,30) \\
10(8,20) \\
40(32,78)\end{array}$ \\
\hline esc & Escolaridade & $\begin{array}{l}\text { 1: Fund. incompleto } \\
\text { 2: Fund. completo } \\
\text { 3: Médio incompleto } \\
\text { 4: Médio completo } \\
\text { 5: Sup. incompleto } \\
\text { 6: Sup. completo } \\
\text { 7: Pós-graduado }\end{array}$ & $\begin{array}{c}45(36,88) \\
9(7,38) \\
10(8,20) \\
41(33,60) \\
5(4,10) \\
10(8,20) \\
2(1,64)\end{array}$ \\
\hline renda & Renda & $\begin{array}{l}\text { 1: menor que } 1 \text { salário } \\
\text { 2: } 1 \text { salário } \\
\text { 3: entre } 1 \text { e } 2 \text { salários } \\
\text { 4: entre } 2 \text { e } 3 \text { salários } \\
\text { 5: entre } 3 \text { e } 4 \text { salários } \\
\text { 6: maior que } 4 \text { salários }\end{array}$ & $\begin{array}{c}42(34,43) \\
39(31,97) \\
24(19,66) \\
6(4,92) \\
7(5,74) \\
4(3,28)\end{array}$ \\
\hline Dom1 & Domínio físico & Contínua, de 4 a 20 , de acordo com a escala Whoqol HIV bref & $14,82( \pm 3,40)^{*}$ \\
\hline Dom2 & Domínio psicológico & Contínua, de 4 a 20 , de acordo com a escala Whoqol HIV bref & $14,79( \pm 2,66)^{*}$ \\
\hline Dom3 & Independência funcional & Contínua, de 4 a 20 , de acordo com a escala Whoqol HIV bref & $13,40( \pm 2,93)^{*}$ \\
\hline Dom4 & Relações sociais & Contínua, de 4 a 20 , de acordo com a escala Whoqol HIV bref & $14,22( \pm 3,55)^{*}$ \\
\hline Dom5 & Meio ambiente & Contínua, de 4 a 20 , de acordo com a escala Whoqol HIV bref & $12,84( \pm 2,74)^{*}$ \\
\hline Dom6 & Espiritualidade & Contínua, de 4 a 20 , de acordo com a escala Whoqol HIV bref & $15,00( \pm 3,70)^{*}$ \\
\hline
\end{tabular}

*Média 士: Desvio padrão; \%: Frequência; estágio: estágio do HIV; esc: escolaridade; renda: renda mensal; Dom1: Domínio 1 da escala Whoqol HIV bref; Dom2: Domínio 2 da escala Whoqol HIV bref; Dom3: Domínio 3 da escala Whoqol HIV bref; Dom4: Domínio 4 da escala Whoqol HIV bref; Dom5: Domínio 5 da escala Whoqol HIV bref; Dom6: Domínio 6 da escala Whoqol HIV bref 
Todas as variáveis apresentaram uma boa convergência para a construção das variáveis latentes, exceto a renda, conforme dispõe a Figura 1.

O indicador WRMR apresentou-se satisfatório para o modelo proposto, e os índices CFI, TLI e qui-quadrado apresentaram-se ligeiramente abaixo do esperado, já o RMSEA e o intervalo de confiança de $90 \%$ do RMSEA mantiveram-se acima dos valores satisfatórios, conforme aponta a Tabela II.

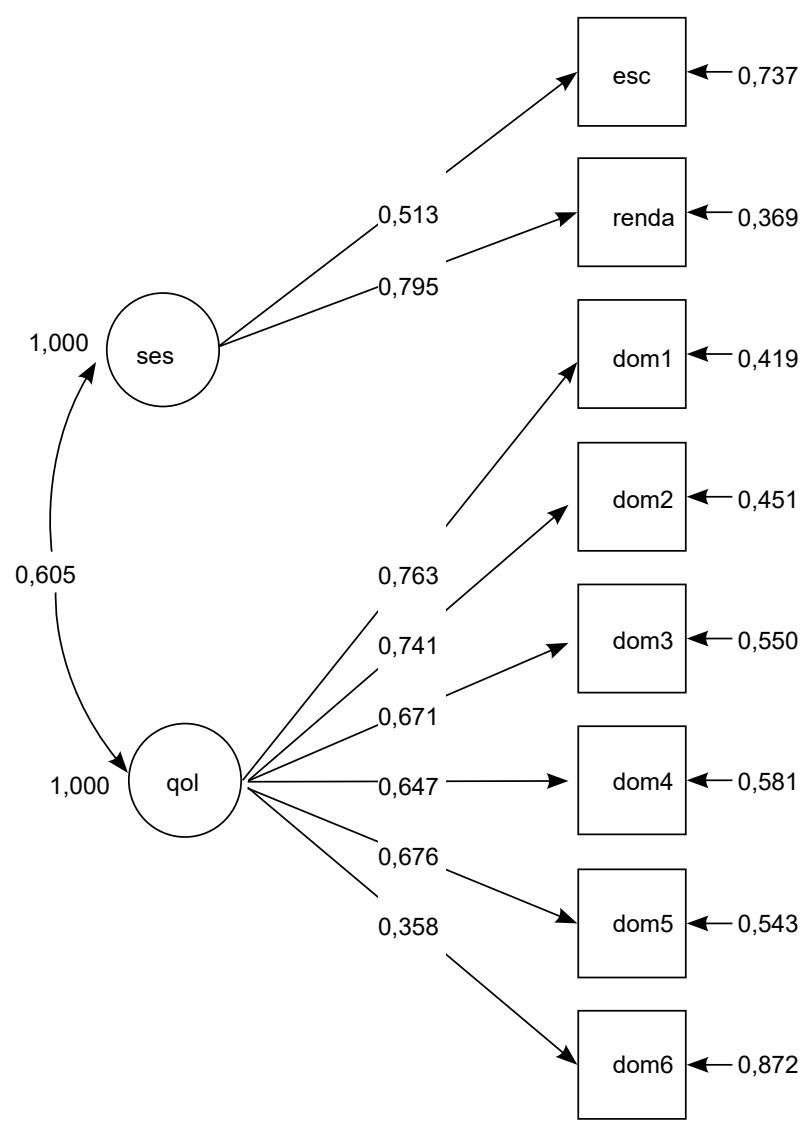

Figura 1 - Análise fatorial confirmatória das variáveis latentes e validade divergente. São Luís, Maranhão, Brasil, 2016-2017.

Siglas: SES: situação socioeconômica; QoL: qualidade de vida; esc: escolaridade; renda: renda mensal; dom1 a dom7: domínios da escala WooQol-Bref-HIV

Tabela II - Resultados esperados e encontrados dos índices de ajustes. São Luís, Maranhão, Brasil, 2016-2017.

\begin{tabular}{lcc}
\hline Índices de ajustes & Valores esperados & Valores encontrados \\
\hline Qui-quadrado/ $\mathbf{p}$ valor & $p$ valor $>0,05$ & $<0,001$ \\
RMSEA & $<0,05$ & 0,117 \\
IC de $\mathbf{9 0 \%}$ do RMSEA & Limite superior $<0,08$ & $0,084-0,153$ \\
CFI & $>0,95$ & 0,858 \\
TLI & $>0,95$ & 0,795 \\
WRMR & $>0,95$ & $\mathbf{0 , 9 8 9}$ \\
\hline
\end{tabular}

RMSEA (Root Mean Square Error of Aproximation); CFI (Comparative Fit Index); TLI (Tucker-Lewis Index); WRMR (Weighted Root Mean Square Residual); IC: Intervalo de Confiança

Todas as cargas fatoriais das variáveis latentes para os indicadores são significantes $(<0,05)$. A maior carga fatorial para o constructo situação socioeconômica (SES) apresentou-se para a variável renda $(0,812)$. Já a maior carga fatorial para o constructo QoL está no domínio físico $(0,765)$. O efeito da espiritualidade na QoL dos indivíduos soropositivos é mediano $(0,358)$. 
Verificou-se, pela inspeção dos coeficientes de determinação $\left(R^{2}\right)$, que $65,9 \%$ da variável renda é explicada pelo status socioeconômico e apenas 12,8\% da espiritualidade é explicada pela QoL (Figura 2).

Em relação à parte estrutural do modelo, o maior efeito observado foi da SES sobre a QoL: para cada variação de um desvio padrão na SES, há um aumento de 0,601 desvios padrão na QoL de indivíduos HIV positivo. Não houve efeito direto significante do estágio do HIV na QoL $(p=0,165)$, nem do SES no estágio $(p=0,610)$. Também não houve efeito indireto do SES na QoL quando mediado pelo estágio do HIV $(p=0,654)$.

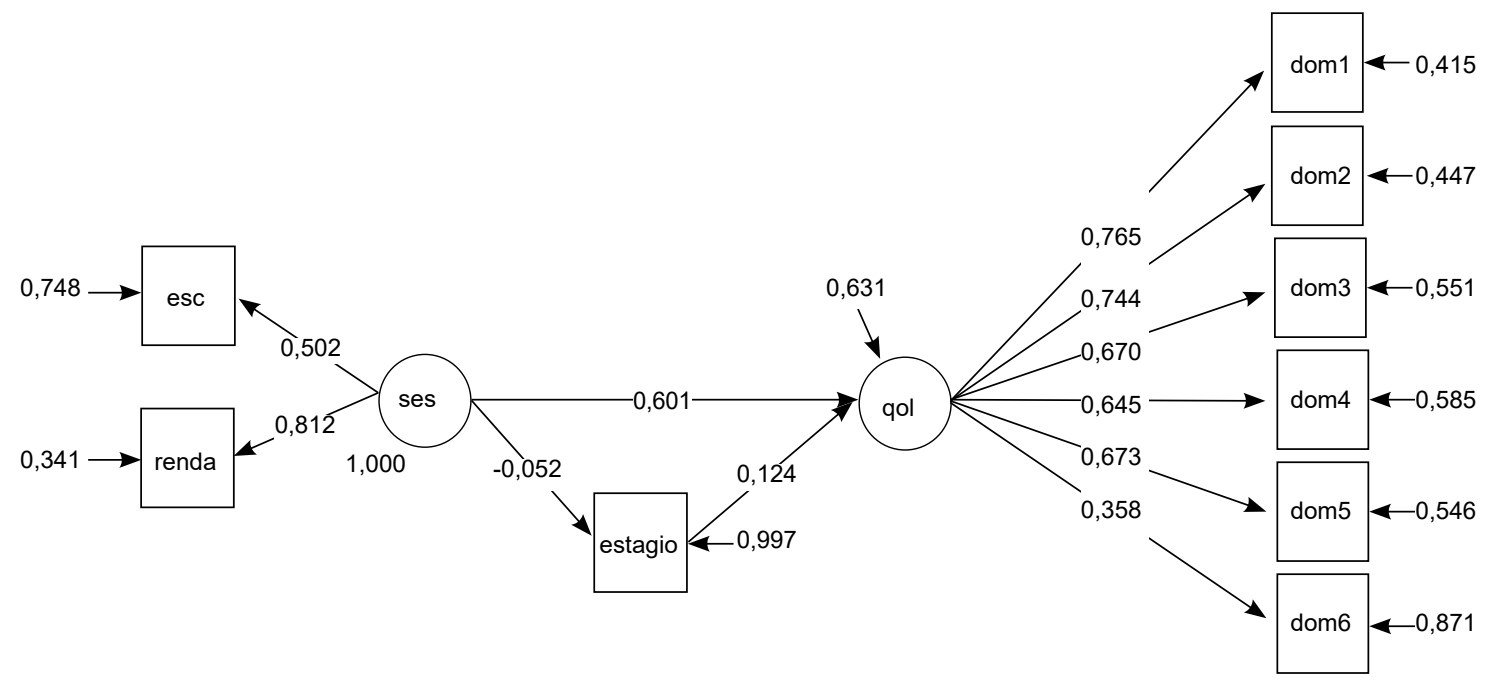

Figura 2 - Modelo estrutural com suas cargas fatoriais e coeficientes de associação entre a situação socioeconômica (SES), o estágio do HIV (Estágio) e a qualidade de vida (QoL). São Luís, Maranhão, Brasil, 2016-2017.

Siglas: estagio: estágio do HIV; esc: escolaridade; renda: renda mensal; Dom1: Domínio 1 da escala Whoqol HIV bref; Dom2: Domínio 2 da escala Whoqol HIV bref; Dom3: Domínio 3 da escala Whoqol HIV bref; Dom4: Domínio 4 da escala Whoqol HIV bref; Dom5: Domínio 5 da escala Whoqol HIV bref; Dom6: Domínio 6 da escala Whoqol HIV bref

Tabela III - Coeficientes padronizados e não padronizados do modelo de equação estrutural. São Luís, Maranhão, Brasil, 2016-2017.

\begin{tabular}{lcc}
\hline Efeitos & $\begin{array}{c}\text { Coeficientes padronizados } \\
\text { / p valor }\end{array}$ & $\begin{array}{c}\text { Coeficientes não padronizados } / \\
\text { p valor }\end{array}$ \\
\hline Qual. de vida $\leftarrow$ Status socioeconom. & $\mathbf{0 , 6 0 1 / < 0 , 0 0 1}$ & $\mathbf{1 , 7 8 1 / < 0 , 0 0 1}$ \\
Qual. de vida $\leftarrow$ Estágio & $0,124 / 0,165$ & $0,241 / 0,189$ \\
Estágio $\leftarrow$ Status socioeconômico & $-0,052 / 0,610$ & $-0,079 / 0,616$ \\
Escolaridade $\leftarrow$ Status socioeconômico & $0,502 /<0,001$ & $1 /-$ \\
Renda $\leftarrow$ Status socioeconômico & $0,812 /<0,001$ & $1,214 / 0,001$ \\
Domínio físico $\leftarrow$ Qual. de vida & $0,765 /<0,001$ & $1 /--$ \\
Domínio psicológico $\leftarrow$ Qual. de vida & $0,744 /<0,001$ & $0,760 /<0,001$ \\
Domínio independência $\leftarrow$ Qual. de vida & $0,670 /<0,001$ & $0,755 /<0,001$ \\
Domínio relações sociais $\leftarrow$ Qual. de vida & $0,645 /<0,001$ & $0,879 /<0,001$ \\
Domínio Meio ambiente $\leftarrow$ Qual. de vida & $0,673 /<0,001$ & $0,709 /<0,001$ \\
Domínio espiritualidade $\leftarrow$ Qual. de vida & $0,358 /<0,001$ & $0,510 /<0,001$ \\
\hline
\end{tabular}

Qual. de vida: Qualidade de vida; socioeconom: socioeconômico

\section{DISCUSSÃO}

No presente estudo, o estágio do HIV não influenciou diretamente a QoL dos indivíduos soropositivos e o efeito total do estágio do HIV na QoL não foi significante. Desse modo, o estágio não esteve associado à ocorrência de diferenças na QoL desses indivíduos. A TARV fornecida pelo Sistema Único de Saúde (SUS) para essa população vem contribuindo para uma melhor QoL, mesmo em casos diagnosticados tardiamente, o que pode explicar essa não associação(18). 
Um estudo comparativo realizado com militares nigerianos observou que a QoL geral é menor entre os soropositivos em comparação com militares soronegativos. Os soropositivos relataram um número maior de eventos negativos na vida e sintomas de trauma (transtorno de estresse pós-traumático), e esses sintomas são um contribuinte significativo para a QoL ${ }^{(19)}$. Além disso, mineradores sul-africanos HIV+ apresentaram uma pontuação significativamente menor na Qol em comparação com os trabalhadores HIV-(20).

Em um estudo que analisou a QoL de PVHA, observou-se uma relação entre maior satisfação com a saúde e QoL, bem como diferenças estatisticamente significativas entre seus domínios e o tempo de diagnóstico, apontando que o tempo do diagnóstico da infecção pelo HIV pode reconfigurar a percepção da QoL ${ }^{(21)}$.

Ressalta-se que os achados da presente investigação apontam uma baixa situação socioeconômica associada a uma baixa QoL dos participantes. Esse resultado está de acordo com outro trabalho, no qual se observou a associação entre uma baixa QoL de PVHA com um baixo nível de escolaridade ${ }^{(22)}$. Quando os autores analisaram os domínios separadamente, observaram-se associações estatisticamente significantes entre um menor nível de escolaridade e baixa QoL no domínio psicológico e meio ambiente, bem como no diagnóstico de infecção pelo HIV nos últimos cinco anos, e baixo escore no domínio das relações sociais ${ }^{(22)}$.

Outro estudo(23) apontou que os escores de QoL não diferiram significativamente entre PVHA que iniciaram a TARV há mais de 5 anos e os indivíduos HIV negativos na Zâmbia e na África do Sul. No entanto houve diferença na QoL entre PVHA que haviam iniciado a TARV há menos de 5 anos e os HIV negativos na Zâmbia, o que aponta novamente o impacto da TARV na melhora da QoL desses indivíduos.

Um estudo recente observou níveis mais elevados na QoL em PVHA com renda superior a dois salários mínimos, tempo maior de diagnóstico de HIV, em uso de TARV e que relataram efeitos colaterais decorrentes dela, assim como os indivíduos clinicamente assintomáticos ${ }^{(24)}$.

A resiliência parece ser um forte componente da QoL e do bem-estar, relacionada à melhoria da saúde física e mental, e acredita-se que melhora com a idade ${ }^{(25)}$. Todavia um estudo ${ }^{(26)} \mathrm{com}$ PVHA verificou que, à medida que a idade avançava, os indivíduos estudados apresentavam baixa resiliência. Outra investigação( ${ }^{(27)}$ observou, também, que idosos com HIV apresentaram baixa QoL quando comparados à jovens vivendo com HIV.

Embora não tenha sido objeto deste estudo investigar diferenças da QoL pelo sexo, as mulheres HIV+ parecem apresentar menos QoL, entretanto uma investigação ${ }^{(28)}$, ao analisar a influência do sexo biológico na QoL de indivíduos com HIV, concluiu que mulheres HIV+ com melhor suporte social relataram melhor QoL geral. Fatores potencialmente modificáveis, como o apoio social e os comportamentos de autocuidado, estão fortemente associados à QoL, sugerindo uma oportunidade para melhorar a vida das $\mathrm{PVHA}^{(28)}$.

Do ponto de vista socioeconômico, um estudo apontou baixa escolaridade e menor renda como fatores diretamente associadas à maior taxa de infecção por $\mathrm{HIV}^{(29)}$. Na QoL, alguns estudos evidenciaram o domínio psicológico como o mais afetado após a infecção(18,30,31). Esses achados diferem dos achados da presente investigação, pois o domínio meio ambiente afetou fortemente a QoL dos participantes.

A literatura, no passado, sugeria uma associação negativa de sintomas e efeitos colaterais com a QoL e o nível de saúde de $\mathrm{PVHA}^{(32)}$. Contudo uma avaliação criteriosa da participação dos efeitos colaterais na variação dos níveis de saúde não tinha sido conduzida até então. Uma investigação ${ }^{(24)}$ trouxe algumas contribuições desses efeitos no status de saúde, como a baixa produtividade no trabalho e a utilização de recursos de saúde devido a irregularidade do ciclo menstrual, icterícia, pancreatite, convulsões e cálculos renais ${ }^{(3)}$.

Apesar de não ter sido verificada essa diferenciação neste estudo, é importante enfatizar que, mesmo sendo tratada e obtendo melhoria das condições de saúde devido à terapia antirretroviral, presume-se que pessoas sintomáticas podem apresentar mais intercorrências, desconfortos e debilidade física quando comparadas com assintomáticas $^{(34)}$.

Outros estudos sugeriram que as PVHA experimentam uma ampla gama de efeitos colaterais devido a TARV e, como consequência, baixa QoL ${ }^{(35,36)}$. Na presente investigação, a SES afetou mais a QoL das PVHA do que o próprio estágio do HIV.

Convergindo com os resultados do presente estudo, um artigo recente observou que uma PVHA sintomática não necessariamente possui menos QoL, o que se deve ao acesso universal ao tratamento e acompanhamento multiprofissional( ${ }^{(9)}$. Isto pode ser explicado pelos novos medicamentos inseridos no protocolo nos últimos anos, que reduziram drasticamente os efeitos colaterais ${ }^{(37)}$.

O presente estudo apresenta algumas limitações que, no entanto, não diminuem sua contribuição. A principal limitação é que os resultados não podem ser generalizados, tendo em vista que o universo estudado é representado 
por uma população de apenas uma unidade de saúde, porém a unidade é considerada um centro de referência para o tratamento de pacientes com HIVIAIDS.

O delineamento transversal e a amostra de conveniência podem ter contribuído para que não se verificasse associação entre o estágio do HIV e a QoL, porém este estudo apresenta pontos fortes, como a aplicação do questionário validado para mensurar QoL e a construção de variáveis latentes para a QoL e a situação socioeconômica, diminuindo possíveis vieses de mensuração e a análise de mediação com MEE.

\section{CONCLUSÃO}

O estágio clínico não se associou à qualidade de vida e não apresentou efeito mediador entre a situação socioeconômica e a qualidade de vida. A situação socioeconômica influenciou diretamente a qualidade de vida de pessoas soropositivas investigadas.

\section{CONFLITOS DE INTERESSE}

Os autores declaram não haver conflitos de interesses.

\section{CONTRIBUIÇÕES}

João Ferreira Silva Junior contribuiu com a elaboração e o delineamento do estudo; a aquisição, análise e interpretação dos dados; e a redação do manuscrito. Carlos Martins Neto e Elisa Miranda Costa contribuíram com a aquisição, análise e interpretação dos dados; e a redação do manuscrito. Bruno Luiz Avelino Cardoso contribuiu com a elaboração e o delineamento do estudo; e a redação do manuscrito. Olga Lorena Maluf Guará Beserra contribuiu com a elaboração e o delineamento do estudo e a aquisição, análise e interpretação dos dados.

\section{FINANCIAMENTO}

A pesquisa contou com o auxílio financeiro concebido pela Fundação de Amparo à Pesquisa e ao Desenvolvimento Científico e Tecnológico do Maranhão (FAPEMA) referente ao Edital TECS-011879/15.

\section{REFERENNCIAS}

1. Abati PAM, Segurado AC. HIV testing and clinical status upon admission to a specialized health care unit in Pará, Brazil. Rev Saúde Pública [Internet]. 2015 [acesso em 2019 Ago 20];49:16. doi: 10.1590/S00348910.2015049004625

2. Melo EA, Maksud I, Agostini R. Cuidado, HIV/Aids e atenção primária no Brasil: desafio para a atenção no Sistema Único de Saúde? Rev Panam Salud Publica [Internet]. 2018 [acesso em 2019 Ago 20];42:e151. doi: 10.26633/RPSP.2018.151

3. Ministério da Saúde (BR), Secretaria de Vigilância em Saúde. Boletim Epidemiológico - HIV AIDS 2018 [Internet]. 2018 [acesso em 2019 Ago 20];49(53). Disponível em: http://www.aids.gov.br/system/tdf/ pub/2016/66196/boletim_hiv_aids_12_2018.pdf?file=1\&type=node\&id=66196\&force $=1$

4. Justice AC, Erlandson KM, Hunt PW, Landay A, Miotti P, Tracy RP. Can Biomarkers Advance HIV Research and Care in the Antiretroviral Therapy Era? J Infect Dis [Internet]. 2018 [acesso em 2019 Ago 20];217(4):5218. doi: 10.1093/infdis/jix586

5. Ministério da Saúde (BR), Secretaria de Vigilância em Saúde, Departamento de Vigilância, Prevenção e Controle das Infecções Sexualmente Transmissíveis, do HIV/Aids e das Hepatites Virais. Manejo da infecção pelo HIV em adultos [Internet]. Brasília: Ministério da Saúde; 2018 [acesso em 2019 Ago 20]. Disponível em: http://www.aids. gov.br/system/tdf/pub/2016/64484/pcdt_adulto_12_2018_web.pdf?file=1\&type=node\&id=64484\&force=1

6. Bradley SM, Rumsfeld JS, Ho PM. Incorporating Health Status in Routine Care to Improve Health Care Value. Jama [Internet]. 2016 [acesso em 2019 Nov 11];316(5):487-8. Disponível em: https://jamanetwork.com/ journals/jama/article-abstract/2540409

7. UNAIDS. Knowledge is power: Know your status, know your viral load. United Nations Program HIVIAIDS 
[Internet]. 2018 [acesso em 2019 Ago 20]. Disponível em: https://www.unaids.org/sites/default/files/media_ asset/jc2940_knowledge-is-power-report_en.pdf

8. Silveira RCW, Lazzarotto AR. A qualidade de vida de pessoas vivendo com HIVIAIDS: Estudo transversal com as organizações não governamentais [Internet]. In: Anais da Semana Científica da Unilasalle; 2018 Out 22-27; Canoas, Brasil. Canoas: Universidade La Salle; 2018 [acesso em 2019 Set 10]. Disponível em: https:// anais.unilasalle.edu.br/index.php/sefic2018/article/download/948/891

9. Machado IK, Luz PM, Lake JE, Castro R, Velasque L, Clark JL, et al. Self-rated health and substance use among individuals in HIV care in Rio de Janeiro, Brazil: a cross-sectional study. Int J STD AIDS [Internet]. 2017 [acesso em 2019 Ago 02];28(12):1175-83. doi: 10.1177\%2F0956462417692278

10. Teixeira PR, Teodorescu LL. Histórias da aids no Brasil 1983 - 2003 [Internet]. 2015 [acesso em 2019 Ago 02]. Disponível em: https://unesdoc.unesco.org/ark:/48223/pf0000235557

11. Lacerda JS, Paulo RG, Aoyama EA, Rodrigues GMM. Evolução medicamentosa do HIV no Brasil desde o AZT até o coquetel disponibilizado pelo Sistema Único de Saúde. Rev Bras Interdisciplin Saude [Internet]. 2019 [acesso em 2020 Mar 11];1(4):83-91. Disponível em: http://revista.rebis.com.br/index.php/rebis/article/ view/247/87

12. Costa MAR, Teston EF, Spigolon DN, Dias LO, Soares CC. Qualidade de Vida sob a ótica de portadores de HIVIAIDS: perspectivas futuras nas práticas educativas. J Res Fundam Care [Internet]. 2019 [acesso em 2020 Mar 11];11(5):1326-32. doi: 10.9789/2175-5361.2019.v11i5.1326-1332

13. Ministério da Saúde (BR), Secretaria de Vigilância em Saúde, Departamento de Vigilância, Prevenção e Controle das Infecções Sexualmente Transmissíveis, do HIVIAids e das Hepatites Virais. Manejo da infecção pelo HIV na Atenção Básica [Internet]. 2015 [acesso em 2020 Mar 13]. Disponível em: http://www.aids.gov.br/ pt-br/pub/2016/o-manejo-da-infeccao-pelo-hiv-na-atencao-basica-para-profissionais-medicos

14. Zimpel RR, Fleck MP. Quality of life in HIV-positive Brazilians: application and validation of the WHOQOL-HIV, Brazilian version. AIDS Care [Internet]. 2007 [acesso em 2019 Ago 02];19(7):923-30. doi: 10.1080/09540120701213765

15. Oliveira BLCA, Silva AM, Rodrigues LS, Rêgo AS. O uso da modelagem com equações estruturais na análise da influência da cor/raça e status socioeconômico na saúde de idosos brasileiros. Rev Bras Cienc Saúde [Internet]. 2016 [acesso em 2019 Set 09];20(2):149-56. Disponível em: https://www.periodicos.ufpb.br/index. php/rbcs/article/view/23106

16. Keith TZ. Multiple Regression and Beyond: an introduction to multiple regression and structural equation modeling. 3th ed. [Londres]: Routledge; 2019.

17. Kline RB. Principles and practice of structural equation modeling. 4th ed. New York: Guilford Press; 2015.

18. Okuno MFP, Gomes AC, Belasco D Jr, Meazzini L, Scherrer G Jr, Belasco AGS. Qualidade de vida de pacientes idosos vivendo com HIVIAIDS. Cad Saude Publica [Internet]. 2014 [acesso em 2019 Set 10];30(7):1551-9. doi: 0.1590/0102-311X00095613

19. Olley BO, Bolajoko AJ. Psychosocial determinants of HIV-related quality of life among HIV-positive military in Nigeria. Int J STD AIDS [Internet]. 2008 [acesso em 2019 Ago 19];19(2):94-8. doi: 10.1258/ijsa.2007.007134

20. Gow J, George G, Govender K. A comparison of quality of life between HIV positive and negative diamond miners in South Africa. Sahara J [Internet]. 2013 [acesso em 2019 Ago 13];10(2):89-95. doi: 10.1080/17290376.2013.870066

21. Hipolito RL, Oliveira DC, Costa TL, Marques SC, Pereira ER, Gomes AMT. Quality of life of people living with HIVIAIDS: temporal, socio-demographic and perceived health relationship. Rev Lat Am Enferm [Internet]. 2017 [acesso em 2019 Ago 11];25:e2874. doi: 10.1590/1518-8345.1258.2874

22. Silva J, Bunn K, Bertoni RF, Neves OA, Traebert J. Quality of life of people living with HIV. AIDS Care [Internet]. 2013 [acesso em 2019 Set 29];25(1):71-6. doi: 10.1080/09540121.2012.686594

23. Thomas R, Burger R, Harper A, Kanema S, Mwenge L, Vanqa N, et al. Differences in health-related quality of life between HIV-positive and HIV-negative people in Zambia and South Africa: a cross-sectional baseline survey of the HPTN 071 (PopART) trial. Lancet Glob Health [Internet]. 2017 [acesso em 2019 Ago 28];5(11):e1133-41. doi: 10.1016/S2214-109X(17)30367-4 
24. Cecilio HPM, Oliveira DC, Oliveira DS, Domingues JP, Marques SC. Quality of life of people living with HIV. Cienc Cuid Saude [Internet]. 2018 [acesso em 2019 Mar 10];17(4):1-8. doi: 10.4025/cienccuidsaude.v17i4.45032

25. Araújo LF, Leal BS, Santos JVO, Sampaio AVC. Análise da resiliência entre pessoas que vivem com HIV/ AIDS: um estudo psicossocial. Psicol Teor Pesqui [Internet]. 2019 [acesso em 2020 Mar 13];35:e35416. doi: 10.1590/0102.3772e35416

26. McGowan JA, Brown J, Lampe FC, Lipman M, Smith C, Rodger A. Resilience and Physical and Mental WellBeing in Adults with and Without HIV. AIDS Behav [Internet]. 2018 [acesso em 2019 Mar 05];22(5):1688-98. doi: 10.1007/s10461-017-1980-6

27. Moore RC, Fazeli PL, Jeste D V, Moore DJ, Grant I, Woods SP. Successful Cognitive Aging and HealthRelated Quality of Life in Younger and Older Adults Infected with HIV. AIDS Behav [Internet]. 2014 [acesso em 2019 Mar 05];18(6):1186-97. doi: 10.1007/s10461-014-0743-X

28. Gielen AC, McDonnell KA, Wu AW, O'Campo P, Faden R. Quality of life among women living with HIV: the importance violence, social support, and self care behaviors. Soc Sci Med [Internet]. 2001 [acesso em 2019 Mar 05];52(2):315-22. doi: 10.1016/S0277-9536(00)00135-0

29. Masquillier C, Wouters E, Loos J, Nöstlinger C. Measuring health-related quality of life of HIV-positive adolescents in resource-constrained settings. PLoS One [Internet]. 2012 [acesso em 2019 Mar 05];7(7):e40628. doi: 10.1371/journal.pone.0040628

30. Shan D, Ge Z, Ming S, Wang L, Sante M, He W, et al. Quality of life and related factors among HIV-positive spouses from serodiscordant couples under antiretroviral therapy in Henan Province, China. PLoS One [Internet]. 2011 [acesso em 2019 Ago 02];6(6):e21839. doi: 10.1371/journal.pone.0021839

31. Desrosiers A, Blokhina E, Krupitsky E, Zvartau E, Schottenfeld R, Chawarski M. Psychiatric symptoms, quality of life, and HIV status among people using opioids in Saint Petersburg, Russia. Drug Alcohol Depend [Internet]. 2017 [acesso em 2019 Ago 02];1(172):60-5. doi: 10.1016/j.drugalcdep.2016.12.007

32. Lindayani L, Ibrahim K, Wang JD, Ko NY. Independent and synergistic effects of self- and public stigmas on quality of life of HIV-infected persons. AIDS Care [Internet]. 2018 [acesso em 2019 Ago 02];30(6):706-13. doi: 10.1080/09540121.2017.1396282

33. DiBonaventura MC, Guptaa S, Chob M, Mrus J. The association of HIVIAIDS treatment side effects with health status, work productivity, and resource use. AIDS Care [Internet]. 2012 [acesso em 2019 Ago 02];24(6):744-55. doi: 10.1080/09540121.2011.630363

34. Seidl EMF, Zannon CMLC, Tróccoli BT. Pessoas Vivendo com HIVIAIDS: Enfrentamento, Suporte Social e Qualidade de Vida. Psicol Reflex Crit [Internet]. 2005 [acesso em 2019 Ago 02];18(2):188-95. doi: 10.1590/ S0102-79722005000200006

35. Ruiz-Pérez I, Olry de Labry A, López-Ruz MA, Del Arco-Jiménez A, Rodríguez-Baño J, Causse-Prados M, et al. Estado clínico, adherencia al TARGA y calidad de vida en pacientes con infección por VIH tratados con antirretrovirales. Enferm Infecc Microbiol Clin [Internet]. 2005 [acesso em 2019 Ago 02];23:581-5. Disponível em: https://www.elsevier.es/es-revista-enfermedades-infecciosas-microbiologia-clinica-28-pdf-13081565

36. Medeiros B, Saldanha A. Religiosidade e qualidade de vida em pessoas com HIV. Estud Psicol [Internet]. 2012 [acesso em 2019 Ago 02];29(1):53-61. doi: 10.1590/S0103-166X2012000100006

37. Lopes MIBF. Tratando HIV no futuro: novas drogas antirretrovirais. Brazilian J Infect Dis [Internet]. 2016 [acesso em 2019 Ago 02];2(3):91-5. Disponível em: https://www.bjid.org.br/en-pdf-X2177511716559704

\author{
Endereço para correspondência: \\ João Ferreira Silva Junior \\ Departamento de Saúde Pública da Universidade Federal do Maranhão \\ Rua Barão de Itapary, 155 \\ Bairro: Centro \\ CEP: 65020-070 - São Luís - MA - Brasil \\ E-mail: jjunior39@yahoo.com.br
}

Como citar: Silva JF Junior, Martins C Neto, Cardoso BLA, Costa EM, Beserra OLMG, Carneiro VS. Qualidade de vida de pessoas soropositivas: relação entre situação socioeconômica e estágio viral. Rev Bras Promoç Saúde. 2020;33:9841. 